\title{
Baller-Gerold Syndrome in a Premature Infant with a Mutation in the RECQL4 Gene
}

\section{Ji Sook Kim, MD}

Department of Pediatrics, Kyungpook National University Children's Hospital, School of Medicine, Kyungpook National University, Daegu, Korea

\section{ABSTRACT}

Baller-Gerold syndrome is a rare autosomal recessive disorder characterized by premature fusion of the cranial sutures and malformation of the upper limb extremities at birth. Although the pathogenesis of Baller-Gerold Syndrome is not fully understood, it is mainly caused by mutations in the RecQ like helicase 4 (RECQL4) gene located on chromosome 8q24.3, which encodes the RECQL4 protein involved in normal DNA replication and repair. This study reports the case of a female premature infant with craniosynostosis of bilateral coronal sutures, resulting in a dysmorphic face and hypoplastic thumbs on both hands at birth, which are consistent with the core characteristics of Baller-Gerold syndrome. Diagnostic whole exome sequencing of the patient revealed a homozygous deletion from exon 13 to 18 in the RECQL4 gene. To the best of my knowledge, this is the first reported case of Baller-Gerold syndrome with RECQL4 gene mutation confirmed by diagnostic whole exome sequencing in Korea.

Key Words: Craniosynostoses; Hand deformities; Infant, premature

\section{INTRODUCTION}

Baller-Gerold syndrome (BGS) is a rare genetic disorder, also referred to as craniosynostosis-radial aplasia syndrome, which was first described independently by Baller ${ }^{1)}$ in 1950 and $\mathrm{Gerold}^{2)}$ in 1959. It is characterized by craniosynostosis, particularly of the coronal sutures, a dysmorphic face, such as a prominent forehead, ocular proptosis, hypertelorism, and a small mouth, and limb abnormalities, especially in the upper extremities, including missing fingers and malformed or absent thumbs ${ }^{3,4}$. In addition to phenotypic variability, additional features presented in BGS include failure to thrive, developmental delay, and systemic anomalies such as congenital heart disease (ventricular septal defect and patent ductus arteriosus), renal agenesis, an imperforate anus, and nervous system abnormalities (agenesis of the corpus callosum, or seizure disorders) $^{5}$. The pattern of inheritance in BGS is thought to be autosomal recessive, and the condition is mainly caused by mutations in the RecQ like helicase 4 (RECQL4) gene located on chromosome 8q24.3, which encodes RecQ helicases ${ }^{6)}$. Here, I report the first Korean case of a premature infant diagnosed as BGS with
Received: 25 July 2019

Revised: 20 September 2019

Accepted: 23 September 2019

Correspondence to: Ji Sook Kim, MD

Department of Pediatrics, Kyungpook National University Children's Hospital, School of Medicine, Kyungpook National University, 807 Hoguk-ro, Buk-gu, Daegu 41404, Korea

Tel: +82-53-200-2750

Fax: +82-53-200-2029

E-mail: jisook.kim.neo@gmail.com

Copyright(c)

By Korean Society of Neonatology.

All right reserved.

This is an Open-Access article distributed under the terms of the Creative Commons Attribution Non-Commercial License (http://creativecommons.org/licenses/ by-nc/4.0), which permits unrestricted non-commercial use, distribution, and reproduction in any medium, provided the original work is properly cited. 
craniosynostosis of the coronal sutures, thumb hypoplasia of both hands, and a mutation in the RECQL4 gene confirmed by diagnostic whole exome sequencing (WES).

\section{CASE REPORT}

A 1-day-old female infant weighing 1,490 g was born at 35 weeks and 1 day of gestational age by repeat cesarean section because of preterm prelabor rupture of the membrane and unconrolled preterm labor. Apgar scores were 7 at 1 minute and 9 at 5 minutes. The pregnancy and delivery were uneventful despite suspected right thumb anomaly on the prenatal ultrasonography. There was no exposure of teratogenic agents, including sodium valproate during pregnancy. A family history of genetic disorders or metabolic diseases was unknown. At birth, her body weight was $1,490 \mathrm{~g}(<10$ th percentile), which was defined as small for the gestational age, height was $37 \mathrm{~cm}(<10$ th percentile), and head circumference was $28.5 \mathrm{~cm}$ ( $<10$ th percentile). Vital signs were as follows: blood pressure, 61/34 mm Hg; heart rate, 141 beats/ minute; respiratory rate, 68 beats/minute; and body temperature, $36.5^{\circ} \mathrm{C}$. Physical examination on admission revealed a palpable ridge on the bilateral coronal sutures, a dysmorphic face, such as a prominent forehead, a high arched palate, and low set ears, a floating thumb on the right hand, and a malformed thumb with a tight web space between the thumb and index finger on the left hand (Figure 1). The limited movement of both elbows, which were not fully extended $\left(130^{\circ}\right)$ was also observed.
Initial blood test results were non-specific. The initial infantogram showed a mild diffuse haziness with interstitial opacities, air bronchograms on both lung fields, and the dislocated joint of both elbows. A few rudimentary bones in both hypoplastic thumbs were detected by a hand X-ray. On the 24th day after birth, serial skull X-rays revealed the early fusion of the coronal sutures. On the 27th day, we confirmed craniosynostosis of the bilateral coronal sutures by three-dimensional (3D) head computed tomography (CT) (Figure 2). Brain ultrasonography, magnetic resonance imaging, echocardiography, and abdominal ultrasonography were performed to evaluate associated anomalies and the results of these were normal.

Genetic testing was conducted to evaluate the cause of multiple anomalies. Peripheral chromosome analysis showed a karyotype of 46, XX, and chromosomal microarray results were normal. To identify genetic disorders, diagnostic WES was performed and a homozygous deletion from exon 13 to 19 in the RECQL4 gene was detected, which was consistent with BGS associated with craniosynostosis and upper limb anomalies at birth. Direct polymerase chain reaction (PCR) and sequencing on the RECQL4 gene (exon 13 to 19) in the patient confirmed a homozygous deletion from exon 13 to 17 and in part of exon 18 in the RECQL4 gene (Figure 3). Direct PCR and sequencing on the RECQL4 gene in the parents and sister were performed for genetic counseling and the results were normal suggesting that the deletion was de novo.

The patient received continuous positive airway pressure (CPAP) therapy because of progressive respiratory distress, including moaning sounds, mild chest retraction, and low oxygen saturation

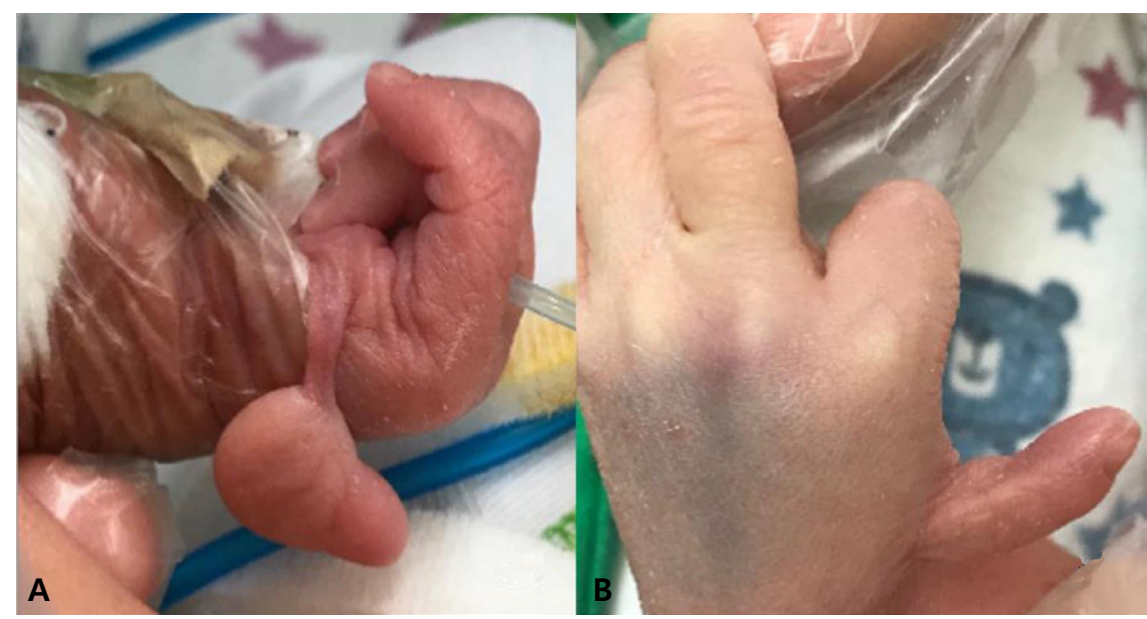

Figure 1. Hypoplastic thumbs on both hands. (A) Floating thumb on the right hand, (B) a malformed thumb with a tight web space between the thumb and index finger on the left hand. 


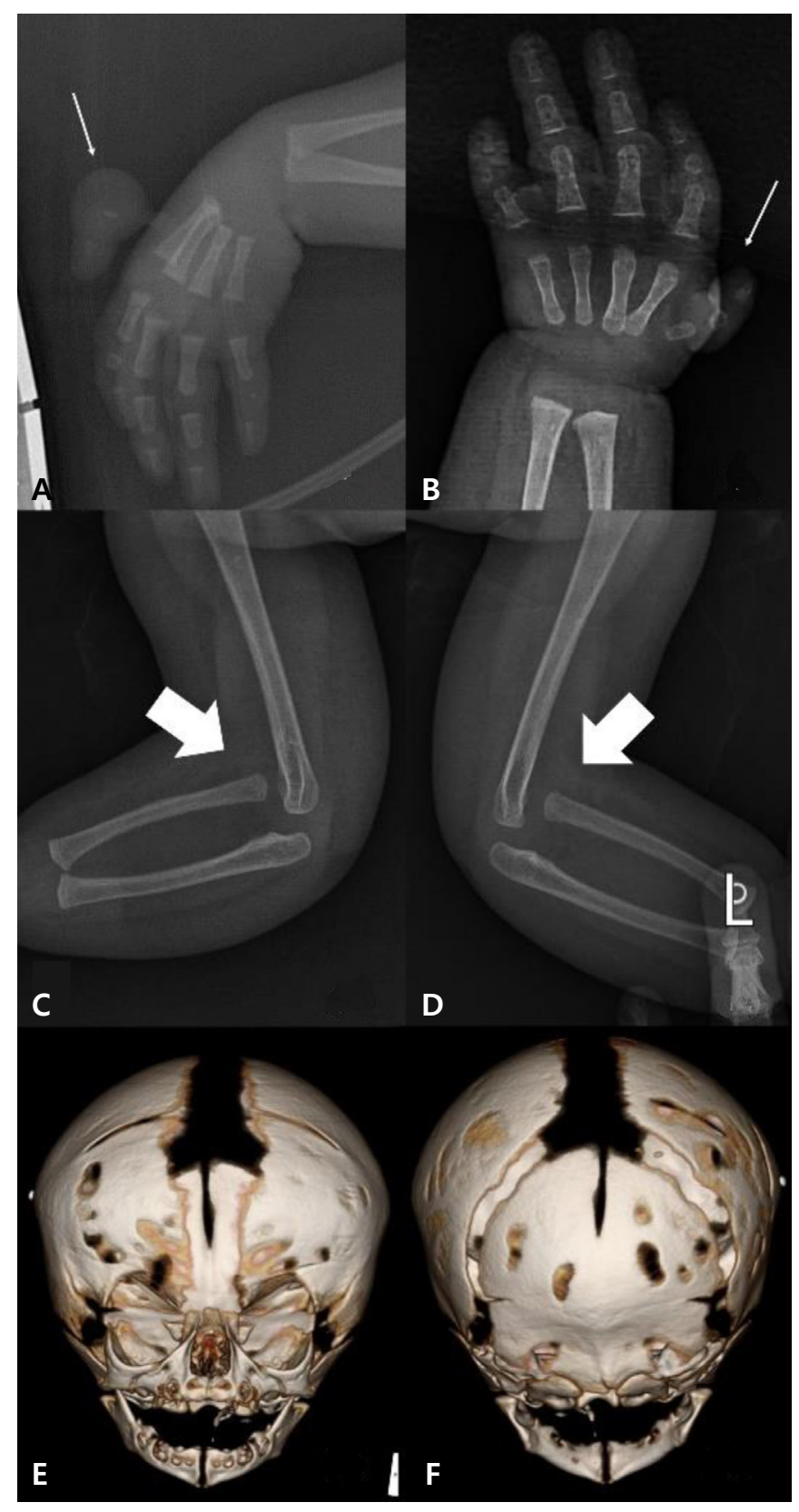

Figure 2. Hand X-ray, elbow X-ray, and three-dimensional (3D)head computed tomography (CT). Hand X-ray showing (A) rudimentary bones in the floating thumb of the right hand and (B) a malformed thumb with tight web of the left hand (narrow arrows), elbow X-ray showing the suspected dislocated joints of the right (C) and left (D) elbows (broad arrows), 3D-head CT showing bilateral premature fusion of coronary sutures (E, F).

( $80 \%$ to $90 \%$ ) at 1 hour after birth, which was diagnosed as mild respiratory distress syndrome (RDS) based on the findings of the initial chest X-ray. On the 5th day after birth, she had no limitation of movement in both elbows, which were fully extended, and the infantogram showed the spontaneous reduction of her dislocated
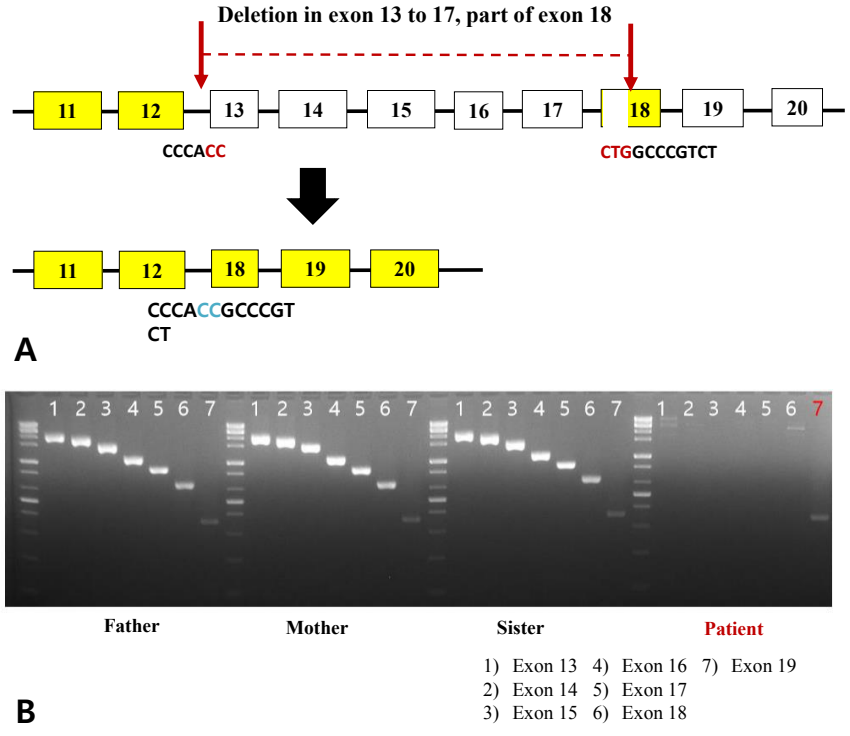

Figure 3. Direct polymerase chain reaction (PCR) and sequencing on the RecQ like helicase 4 (RECQL4) gene (exon 13 to 19) in the patient and their family. (A) Homozygous deletions from exon 13 to 17 and in part of exon 18 in the RECQL4 gene were detected in the patient. (B) The results of direct PCR and sequencing in the parents and sister were normal.

elbow joints. Following the improvement in RDS, she was weaned from CPAP on the 5th day and achieved full enteral feeding on the 9 th day after admission. At 37 weeks of corrected gestational age, her clinical condition was stable with normal growth velocity and she was weaned from the incubator. She was discharged from the neonatal intensive care unit at 41 days after birth without oxygen supplementation and the need for a gastric nutrition tube.

The patient was followed up to monitor growth and develop. mental status, craniosynostosis, and thumb hypoplasia in the outpatient neonatal, pediatric neurologic, neurosurgery, and orthopedic clinics after discharge. On the 125th day of life, she underwent an operation for the removal of the right hypoplastic thumb with necrotic change. At that time, the growth velocity of the head circumference was low (less than $1 \mathrm{~cm}$ per month). In addition, there were palpable ridges on the sagittal and lambdoid sutures on physical examination and progressive premature fusion of all the cranial sutures was confirmed by a follow-up 3D-head CT. At 6 months of corrected gestational age (222 days after birth), she underwent cranioplasty, which consisted of craniotomy and distractor fixation. Currently, the patient is undergoing bone distraction to achieve the desired head shape with regular follow-up to monitor growth and development. At the follow-up at 6 months of corrected gestational age, the patient had not grown 
up to catch-up, her weight $(5.7 \mathrm{~kg})$, height $(60.0 \mathrm{~cm})$, and head circumference $(39.9 \mathrm{~cm})$ were still $<10$ th percentile for the corrected gestational age. However, her development was appropriate for the corrected gestational age. Pollicization of the left hypoplastic thumb would be performed at around 12 months of life.

\section{DISCUSSION}

BGS is a rare, autosomal recessive disorder. The exact prevalence of BGS, which affects approximately less than 1 per million people, is unknown, and fewer than 40 cases have been reported in the medical literature ${ }^{7)}$. Based on previous reports, the key features of BGS include early fusion of the cranial sutures, espe cially coronal sutures, and pre-axial limb malformation (radius or thumbs), which are apparent at birth ${ }^{8)}$. Therefore, BGS is a genetic disorder that should be suspected in neonates with craniosynostosis and upper limb malformation at birth, such as in this case, where premature fusion of the coronal sutures and hypoplastic thumbs were observed.

Craniosynostosis involving any or all sutures is a core feature that can be used to differentiate BGS from other syndromes with upper limb malformation and hematopoietic abnormalities, such as thrombocytopenia-absent radius syndrome and HoltOram syndrome ${ }^{5,9)}$. Although the involvement of the coronal sutures (alone or in combination) is most common (64\%), all cranial sutures may be affected, such as the metopic (36\%), lambdoid (27\%), and sagittal (9\%) sutures ${ }^{8)}$. The clinical severity of craniosynostosis in BGS is variable; plagiocephaly, a prominent forehead, and ocular proptosis may be presented with premature fusion of the cranial sutures. In this case, craniosynostosis of the coronal sutures was immediately detected after birth and progressive premature fusion of all cranial sutures were observed at 3 months of life, which resulted in microcephaly, a prominent forehead, bulging eyes, and shallow orbits.

The defect of pre-axial upper extremities is another major feature of BGS, which has been observed in previous patients. The patient in this case had hypoplastic thumbs on both hands (type II for the left thumb and type IV for the right thumb). The absence or hypoplasia of thumbs is the most common deformity in BGS; it occurs in $95 \%$ of patients, followed by the absence or hypoplasia of radii in $77 \%$ of patients. The defects of upper extremities (excluding the pre-axial portion) include anomalies of the phalanges of fingers and metacarpal bones in $95 \%$ of cases, a short or curved ulnar in $82 \%$ of cases, and fused or absent carpal bones in $27 \%$ of cases ${ }^{5)}$. Various skeletal anomalies have been reported, such as vertebral defects, including scoliosis, missing vertebrae, flat vertebral bodies, and abnormal toes (absent or hypoplastic phalanges of toes). Multiple malformations associated with visceral organs have also been reported in the literature. The visceral defects observed in BGS patients include polymicrogyria, hydrocephalus, corpus callosum agenesis in the central nervous system, ventricular septal defects, tetralogy of Fallot, subaortic valvular hypertrophy in the cardiovascular system, kidney ectopia, hydronephrosis in the urogenital system, and an imperforated anus $^{9-11)}$.

Mutations in the DNA helicase of the RECQL4 gene have been identified mainly in patients with $\mathrm{BGS}^{6)}$. Kaneko et al. ${ }^{4)}$ reported a homozygous deletion from intron 12 to the former part of exon 18 in the RECQL4 gene in three patients with BGS, which was similar to the mutation of the RECQL4 gene detected in my patient with homozygous deletion from exon 13 to 18 in the RECQL4 gene. Mutations in the RECQL4 gene have been associated with two other autosomal recessive disorders: Rothmund-Thomson syndrome (RTS) and RAPADILINO syndrome ${ }^{12,13)}$. Therefore, RTS and RAPADILINO syndrome should be excluded in the diagnosis of BGS based on the distinctive clinical presentation of each disorder. RTS is characterized by poikiloderma, sparse hair in the eyelashes and eyebrows, small stature, skeletal and dental abnormalities, juvenile cataracts, and increased risk of cancers such as osteosarcoma and skin cancer ${ }^{12)}$. RAPADILINO syndrome is characterized by radial ray defects, patellae hypoplasia or aplasia, a cleft or highly arched palate, diarrhea, dislocated joints, a small size, limb malformation, a slender nose, and normal intelligence $^{14)}$. To differentiate BGS from RTS and RAPADILINO syndrome, major symptoms or symptoms essential for the diagnosis of each disorder should be considered. Although the presence of craniosynostosis and radial hypoplasia occurring simultaneously at birth may be a diagnostic criterion for BGS, patients diagnosed as RTS or RAPADILINO syndrome have also been reported to present phenotypes overlapping with those of $\mathrm{BGS}^{13)}$. Further studies are needed to define distinctive features for the diagnosis of BGS, RTS, and RAPADILINO syndrome and to determine whether the three RECQL4-associated diseases may be regarded as separate disorders or belonging to the same clinical spectrum.

The treatment for the symptoms of BGS depends on the clinical manifestation. Craniosynostosis may require surgical treatment 
such as craniotomy, which is determined by neurosurgery or craniofacial specialists. Surgery for bilateral craniosynostosis is recommended before 6 months of age. Our patient underwent craniotomy and distractor fixation at 6 months of corrected age because of the progression of fusion of the coronal sutures. In BGS patients with absent or hypoplastic thumbs, pollicization of the index finger is an appropriate method to restore functional grasp; however, many patients with aplastic or hypoplastic thumbs can grasp objects without orthopedic surgery ${ }^{15)}$. The human DNA helicase, RECQL4, is known to play an important role in DNA replication, transcription, recombination, and repair. Recent biochemical and molecular studies found that mutations in the RECQL4 gene could cause genomic instability and carcinogenesis ${ }^{16)}$. Debeljak et al. ${ }^{17)}$ reported a case where BGS was presented with lymphoma. In addition, pathogenic RECQL4 mutations in patients with RTS may be associated with an increased risk for developing osteosarcoma ${ }^{18)}$. Therefore, BGS patients with pathogenic RECQL4 mutations, such as in this case, should be closely observed for symptoms or signs of malignancies such as osteo. sarcoma and lymphoma.

In conclusion, BGS should be considered in infants with an early fusion of a specific cranial suture and malformation of the upper limb extremities at birth. To differentiate from other genetic disorders with overlapping features, careful physical examination to detect key symptoms or signs for the diagnosis of BGS is necessary and a proper genetic test that includes the RECQL4 gene is needed to confirm BGS. This case report describes a premature infant with BGS presenting with early fusion of the coronal sutures and hypoplastic thumbs at birth and harboring a mutation in the RECQL4 gene. To the best of my knowledge, this is the first case of RECQL4-related BGS in Korea.

\section{ARTICLE INFORMATION}

\section{Ethical statement}

This study was approved by the Institutional Review Board of Kyungpook National University Chilgok Hospital (2019-07-012) and a waiver of consent was granted owing to retrospective chart review without patient contact.

\section{Conflicts of interest}

No potential conflict of interest relevant to this article was reported.

\section{Author contributions}

Conception or design: J.S.K.

Acquisition, analysis, or interpretation of data: J.S.K.

Drafting the work or revising: J.S.K.

Final approval of the manuscript: J.S.K.

\section{ORCID}

Ji Sook Kim https://orcid.org/0000-0002-0100-5845

\section{Acknowledgments}

None

\section{REFERENCES}

1. Baller F. Radiusaplasie und Inzucht. Z Mensch Vererb Konstitutionsl 1950;29:782-90.

2. Gerold M. Healing of a fracture in an unusual case of congenital anomaly of the upper extremities. Zentralbl Chir 1959;84:831-4.

3. Piard J, Aral B, Vabres P, Holder-Espinasse M, Megarbane A, Gauthier S, et al. Search for ReCQL4 mutations in 39 patients genotyped for suspected Rothmund-Thomson/Baller-Gerold syndromes. Clin Genet 2015;87:244-51.

4. Kaneko H, Izumi R, Oda H, Ohara O, Sameshima K, Ohnishi H, et al. Nationwide survey of Baller Gerold syndrome in Japanese population. Mol Med Rep 2017;15:3222-4.

5. Ramos Fuentes FJ, Nicholson L, Scott CI Jr. Phenotypic variability in the Baller-Gerold syndrome: report of a mildly affected patient and review of the literature. Eur J Pediatr 1994; 153:483-7.

6. Van Maldergem L, Siitonen HA, Jalkh N, Chouery E, De Roy M, Delague $\mathrm{V}$, et al. Revisiting the craniosynostosis-radial ray hypoplasia association: Baller-Gerold syndrome caused by mutations in the RECQL4 gene. J Med Genet 2006;43:148-52.

7. Adam MP, Ardinger HH, Pagon RA, Wallace SE. GeneReviews [Internet]. Seattle (WA): University of Washington; 1993-2019. Baller-Gerold syndrome [cited 2019 Nov 12]. Available from: https://www.ncbi.nlm.nih.gov/books/NBK1204.

8. Santos de Oliveira R, Lajeunie E, Arnaud E, Renier D. Fetal exposure to sodium valproate associated with Baller-Gerold syndrome: case report and review of the literature. Childs Nerv Syst 2006;22:90-4.

9. Dallapiccola B, Zelante L, Mingarelli R, Pellegrino M, Bertozzi V. Baller-Gerold syndrome: case report and clinical and radiological review. Am J Med Genet 1992;42:365-8.

10. Boudreaux JM, Colon MA, Lorusso GD, Parro EA, Pelias MZ. Baller-Gerold syndrome: an 11th case of craniosynostosis and 
radial aplasia. Am J Med Genet 1990;37:447-50.

11. Woon KC, Kokich VG, Clarren SK, Cohen MM Jr. Craniosyno stosis with associated cranial base anomalies: a morphologic and histologic study of affected like-sexed twins. Teratology 1980;22:23-35.

12. Megarbane A, Melki I, Souraty N, Gerbaka J, El Ghouzzi V, Bonaventure J, et al. Overlap between Baller-Gerold and Rothmund-Thomson syndrome. Clin Dysmorphol 2000;9:303-5.

13. Siitonen HA, Sotkasiira J, Biervliet M, Benmansour A, Capri Y, Cormier-Daire V, et al. The mutation spectrum in RECQL4 dis eases. Eur J Hum Genet 2009;17:151-8.

14. Siitonen HA, Kopra O, Kaariainen H, Haravuori H, Winter RM, Saamanen AM, et al. Molecular defect of RAPADILINO syn drome expands the phenotype spectrum of RECQL diseases.
Hum Mol Genet 2003;12:2837-44.

15. Foucher G, Medina J, Lorea P, Pivato G. Principalization of pollicization of the index finger in congenital absence of the thumb. Tech Hand Up Extrem Surg 2005;9:96-104.

16. Mo D, Zhao Y, Balajee AS. Human RecQL4 helicase plays multifaceted roles in the genomic stability of normal and cancer cells. Cancer Lett 2018;413:1-10.

17. Debeljak M, Zver A, Jazbec J. A patient with Baller-Gerold syndrome and midline NK/T lymphoma. Am J Med Genet A 2009;149A:755-9.

18. Wang LL, Gannavarapu A, Kozinetz CA, Levy ML, Lewis RA, Chintagumpala MM, et al. Association between osteosarcoma and deleterious mutations in the RECQL4 gene in RothmundThomson syndrome. J Natl Cancer Inst 2003;95:669-74. 